\title{
ENHANCEMENT OF Qos IN LTE DOWNLINK SYSTEMS USING FREQUENCY DIVERSITY SELECTIVITY SCHEDULING
}

\author{
S.Sharmila ${ }^{1}$, C.Veera lakshmi ${ }^{2}$ \\ ${ }^{I} P G$ Student/Dept. of ECE, M.Kumarasamy college of Engineering, Karur, India \\ ${ }^{2}$ Assistant professor/Dept. of ECE, M.Kumarasamy college of Engineering, Karur, India
}

\begin{abstract}
LTE is a broadband wireless access network offers rich suite of multimedia applications and developed by Third generation partnership project is designed to support ubiquitous delivery of multimedia services. For this reason, numerous research groups are annoying to utilize its concert. Scheduling and resource allocations are the vital components of wireless data schemes. The CSI (channel state information) makes possible to acclimatize transmissions to channel conditions, which is essential for achieving reliable communication with high data rates in systems. To assist resource allocation, classification algorithm is used to identify mobility users. According to the taxonomy, Frequency diversity and selectivity scheduling algorithm is proposed to synchronies low mobility user for multi user diversity and high mobility user for frequency diversity. Continuous rate adaptation is used to make best use of the channel spectral efficiency given a limited number of time-frequency resource blocks. The capacity allocation allocates the total capacity among each of the base stations in an amount that corresponds to the predictable traffic load at each base station. It is verified by simulation that throughput and capacity is increased for the proposed algorithm.
\end{abstract}

Keywords-Long term evolution (LTE), OFDMA, Frequency diversity,Multi user diversity,Channel quality indicator,Resource block, Proportional fair scheduling, Channel state information. $* * *$

\section{INTRODUCTION}

Long Term Evolution (LTE) is a radio proposal technology which was used to accomplish superior crest throughputs than High Speed Packet Access .LTE is typical choice for wireless Communication of soaring rapidity data rate for mobile phones with elevated capacity support. It depends on GSM/EDGE system technologies, mounting capacity and speed using a dissimilar radio edge together with interior network improvements. With the mobile networks migrating towards LTE and all-IP networks, people are interested to connect to the Internet anytime, anywhere and from the world. In disparity, OFDM is not a stable covering in modulation methods. The amplitude and time segment of every information or data is stable in each symbol. There can be several large peaks in an OFDM symbol. The Radio Frequency power amplifier must be proficient of achieving peak power and requires a superior speaker to knob a given standard control where extract is not worn Efficiency is therefore lower [1]. To overcome the drawback of OFDM, OFDMA is in use as the multiplexing design in the LTE downlink. 802.11a is the finest technique to portray OFDMA is by distinct it with a packet-tilting networking method. In 802.11a, Carrier-Sense Multiple Access (CSMA) is the multiplexing process. Downlink and uplink traffic from the regular admission point to mobile customer stations is the type of practical apparatus that create LTE for better-quality above $3 \mathrm{G}$ technologies is the well-organized use of OFDMA collective with MIMO well-groomed antenna in uplink and downlink radio transmission. The structural design of LTE is shown in Fig -1

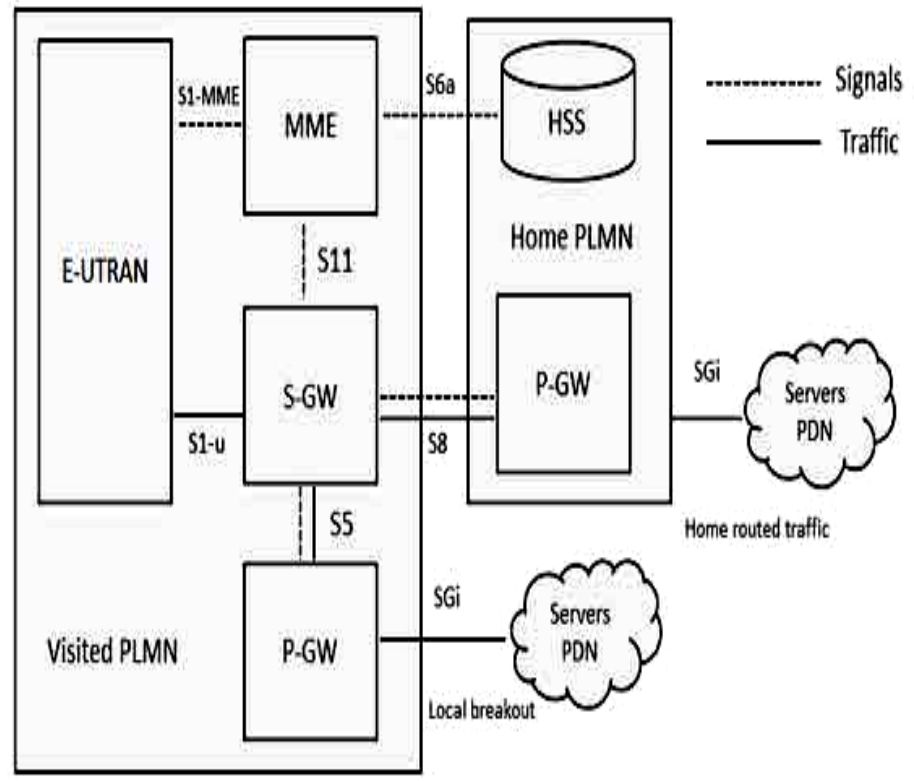

Fig- 1: LTE Architecture

OFDMA is a hopeful applicant because it not only take over OFDM resistance to inter symbol intrusion and frequency 
selective fading ,but also make efficient multi user diversity by performing the channel fading as a channel methods. It supports packet oriented approaches which explains the terms of efficiency and latency. The dissimilarity between OFDM and OFDMA is that OFDMA has the ability to allocate a subset of subcarriers to users, make use of multi-user diversity.

F. Capozzi, G. Piro peak proposed that, the data rates for the downlink is $100 \mathrm{mbps}$ and uplink carries $50 \mathrm{mbps}$ [2].Diversity scheme is a process for improving the stability of a message gesture by using additional communication channels with diverse individuality. Diversity plays a momentous role in fading and co-channel interference and eliminates error bursts. Frequency diversity supplies the signal or certain information using numerous frequency channels or thickens over a extensive spectrum that is exaggerated by frequency-selective fading Arrangement or selection of user is specified as;at any given time the transmitter selects the most excellent user according to the channel behavior of the transmitter and receiver

The remainder of the paper is structured as follows. In section II, explains different accessible scheduling and resource allocation algorithms. Section III addresses the existing FDSS scheduling scheme. Section IV explains the proposed algorithm. Section V evaluates and examines the performance of the continuous rate adaptation algorithm through simulations and finally, conclusion is haggard in section VI.

\section{RELATED WORK}

In this section, we present existing scheduling and resource allocation schemes which are classified based on several factors. The scheduling strategy evaluates the best user to transmit through a specified time period .By instinct; we want to consign resource to users with excellent channel circumstances so the resource can be used more proficiently and make available of fairness or Qos guarantees to every single one customers. For example, allowing only users close to the base station to transmit with high transmission power may result in very high system throughput, but may starve other users. The block diagram of scheduling and resource allocation is shown in Fig-2.

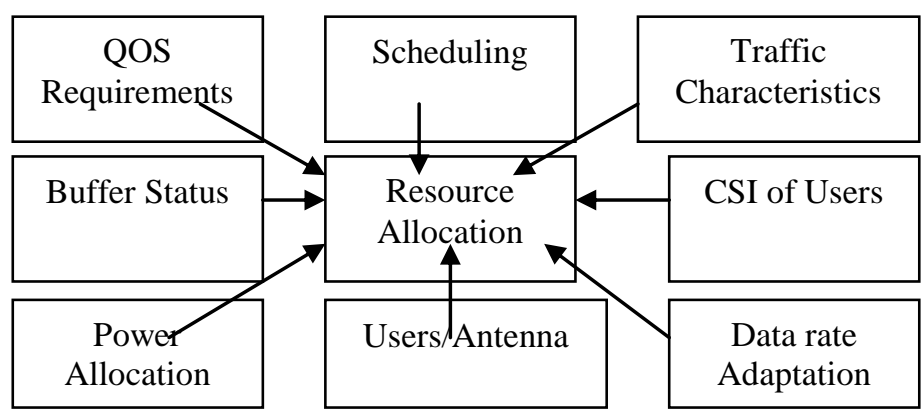

Fig - 2: Scheduling and Resource Allocation
T.Dikamba [3] proposed a scheduling strategy assigns resource blocks to the user with the unsurpassed radio link environment. In sort to execute scheduling, end points propel Channel Quality Indicator (CQI) to the correct foundation location (BS). Fundamentally in the downlink, the BS supplies reference signal (downlink channel) to terminals. These reference signals are worn by user apparatus for the extents of the CQI. A advanced CQI value in a sense it has better channel condition.

S. Husain proposed Round Robin scheduling is a non-attentive scheduling format that lets users take turns in via the collective resources (time/RBs), devoid of taking the on the spot channel conditions into consideration. Consequently, it offers enormous fairness amid the users in radio resource obligation, but reduce the system throughput performance. The improvement of round-robin scheduling is the reduced processing time in view of the fact that the base station does not require to surrender transmission time to notify the users in each block about their owed slot points. On the other hand due to the stochastic temperament of the wireless channel, it is not potential to afford rigid QoS guarantees, i.e., if the channel is in a deep fade it is not probable to accomplish the SINR restriction. In Time and Frequency sphere of influence Round Robin (TFDRR) manifold users are permitted to be scheduled within one TTI in a repeated order Fairness is very important but may have unusual implications in wired and wireless systems. In wired networks, when a convinced quantity of resource is allocated to a user, it is correspondent to conceding the user definite amount of throughput/performance assessment. Conversely, the amount of resource and the performance values are not unswervingly associated J. Fan, Q. Yin [5] proposed a novel resource allocation depends on MCS feedback and considers the realistic limitation in LTE systems that the RB's allocated to the identical user in any specified TTI be obliged to utilize the same MCS were taken in to account; it can be simply functional to the downlink communication of LTE systems. Adaptive resource allocation can achieve significant capacity gain over non adaptive TDMA.H. Kim [6] proposed user mobility prediction methods have been submitted in the literature. These may be worn for resource reservation and service preconfiguration/ altered copy in prospect wireless systems to afford QoS guarantees. Though, our examination of some of these methods by means of deliberate cellular concert shows that these models do not precisely correspond to the mobility patterns of users.

D. Calabuig [7] Dynamic RRM schemes adaptively fine-tune the radio network parameters to the load transfer, place of user, mobility user, QOS requirements, base station density, etc. Dynamic radio resource management methods are measured in the propose of wireless network, which diminish physical cell which is more luxurious.

A. Mills, D. Lister [8] proposed frequency diversity scheduling where the signal is transmitted by means of numerous frequency channels or multiply over a spacious spectrum that is 
exaggerated by frequency-selective fading. The FDS move toward to allocate a user the orthogonal subcarriers far and wide speckled more than an complete frequency band, achieving frequency diversity gain.

\section{FREQUENCY DIVERSITY AND SELECTIVITY SCHEDULING}

The main goal is to allocate the resources to the mobility users, whereas resource allocation is a special case of scheduling. Scheduling for different mobility's i.e high- or low-mobility users has been studied. It has been found that multi-user diversity scheduling is superior for the low-mobility users [10] - [11] while frequency diversity scheduling facilitated the high mobility users [8]. As a result, we develop a novel scheduling algorithm which makes use of both multiuser and frequency diversity (FDSS) simultaneously and the capacity for the mobility users is also determined. To develop FDSS algorithm, User Classification algorithm is used to identify high or low mobility users. This work was done by M.H.Habaebi, J.Chebil [11] proposed that, the CQI techniques were selected and their performance will be calculated in terms of average throughput and Block Error Rate (BLER) for the individual UEs (user equipments) and the overall network.

\subsection{User Classification Algorithm}

A novel user classification algorithm is used to classify highand low-mobility users. Exact speed of the user is not needed for our scheduling [18], only certain speed is needed i.e. mobile speed or a user is with low-mobility or high-mobility. The user mobility classification is not highly complex. By performing field test, cross-over point can be found. Based on the classification, scheduling algorithm is then proposed. If the speed of user is higher than the crossover point called high mobility users and if the speed of user is lower than the crossover point called low mobility users. In LTE only limited feedback information is needed.

The condition for High mobility user is:

$$
\sigma^{2} k>=\sigma^{2} T
$$

The condition for Low mobility user is:

$$
\sigma^{2} \mathrm{k}<=\sigma^{2} \mathrm{~T}
$$

\subsection{FDSS Algorithm}

In frequency diversity, signal is supplied using a number of frequency channels or extend over a large band that is exaggerated by frequency selective fading. In multi-user diversity, many users will be there and according to channel conditions best user is selected. It is also called as frequency selectivity. Frequency diversity and Multi user diversity jointly together called as FDSS algorithm. To identify whether the user is within mobility or not, we need to find the variance which is given by,

$$
\sigma_{\mathrm{k}, \mathrm{m}}^{2}=1 / \mathrm{N}-1 \sum_{\mathrm{n}=1}\left(\mathrm{C}_{\mathrm{k}, \mathrm{m} ., \mathrm{n}}-\mu_{\mathrm{k}, \mathrm{m}}\right)
$$

Where,

$$
\mu_{\mathrm{k}, \mathrm{m}}=1 / \mathrm{N} \sum_{\mathrm{n}=1} \mathrm{C}_{\mathrm{k}, \mathrm{m}, \mathrm{n}}
$$

Denote $\mathrm{C}_{\mathrm{k}, \mathrm{m}, \mathrm{n}}$ to be the reported channel quality indicator (CQI) of user $\mathrm{k}$ for Resource Block Groups $\mathrm{m}$ at transmission time interval (TTI) $n$. If user $k$ is with high mobility, then $C_{k, m}, n$ varies with $\mathrm{n}$ quickly. Otherwise, it changes slowly with TTI (n).Resource block group is that small amount of user is scheduled to a user Resource block group is that small amount of user is scheduled to a user. It has 12 subcarrier in frequency domain and 14 OFDM symbols in time province [19]. Proportional Fairness is used to select the users with respect to instant rate divided by average rate; resulting performance is higher than round robin which allocates a channel in a time slot. Jianwei Huang [13] proposed an algorithm in proportional fair scheduler in the context of high data rate (HDR) system. In the first time slot, the terminals with higher CQI are scheduled. In the second slot of time, the scheduled terminals are cyclically in turn. On the third slot period the process is repeated again alternately [15]. Channel capacity is the good and tightens upper bound on the time of information that can be constantly transmitted over a system. It is specified by,

$$
\mathrm{C}=\mathrm{B} \log _{2}(1+\mathrm{s} / \mathrm{n})
$$

$C$ is measured in bits per second, if the natural logarithm is used; haughty $B$ is in hertz; the signal and noise powers $S$ and $N$ are calculated in watts.

\section{CONTINUOUS RATE ADAPTATION}

In the proposed approach, structure throughput, QoS control, and scheduling justice are jointly incorporated into a framework to energetically execute radio resource-allocation for numerous users, and successfully prefer optimal system parameters such as power and modulation rate to acclimatize to the unreliable channel quality of each resource block. In such systems, frequency-time resources are vigorously shared mutually between users to enhance overall spectral efficiency. At the compassion of the LTE transmission idea is the use of sharedchannel transmission, in which time-frequency resources are animatedly distributed amongst users. This is comparable to the come near to be taken in High-Speed Downlink Packet Access (HSDPA). On the other hand, scheduling in LTE can receive channel variation into account not only in the time field, as with HSPA, but also in the frequency area [21] - [22].

Here, we first concentrate on the optimum power and continuous rate adaptation for solitary resource block of an LTE-based system less than total transmission power limitation 
for the base-station communication. The results are unmitigated to continuous rate adaptation in a multi-user situation under power and portion of time-frequency resource block limitations. The most important objective of this manuscript is to find in cooperation optimum power and continuous-rate in conditions of time-varying and frequency-selective fading channel reaction The scheduler pedals, for every instantaneous of time, to which users the shared resources should be given. It also evaluates the data rate used for every bond. Conversely LTE has, in adding together to the time domain, right to use the resources in frequency field, due to the employ of OFDM technology in the downlink. Consequently the scheduler can, for every one frequency region, select the user with the satisfactory channel conditions. But, scheduling in LTE can achieve channel variation into account not only in the time field, as with HSPA, but also in the frequency area. Every user allocates a number of resource blocks in the time-frequency Since sub-channels and time-slots are independent, the total average data rate of the $\mathrm{j}^{\text {th }}$ user larger than the complete frequency bandwidth B and I time-slots, is equivalent to the amount of standard user data rates in excess of all resource blocks and is specified by,

$$
\overline{\mathbf{R}_{\mathrm{j}}}=\sum_{\mathrm{n}=1}^{\mathrm{N}} \sum_{\mathrm{i}=1}^{\mathrm{I}} \overline{\mathbf{R}_{\mathrm{j}, \mathrm{n}, \mathrm{i}}}(5)
$$

For that reason, over the multi-user system, the scheduling and resource-allocation judgment can be viewed as deciding a rate $\mathrm{R}_{\mathrm{j}}=(\mathrm{R} 1, \mathrm{R} 2 \ldots \mathrm{RJ})$. The method starts by allocating a percentage $\left(x_{\mathrm{jni}}\right)$ of the initial sub-carrier $(n=1)$ in time-slot $(i$ $=1$ ) to $J$ users. This then followed for each and every one subcarriers surrounded by the same time-slot. When a proportion of sub-carriers in the ith time-slot are allocated to every user, this progression iterates over the enduring time-slots.

\section{PERFORMANCE ANALYSIS}

The simulation model is implemented by using MATLAB tool. The Typical Urban channel model has been fraction of the toolbox for researchers and developers in the wireless announcement in view of the fact that the before years of GSM. This fastidious representation was depend on radio channel capacity was demonstrated, used to conclude the require and performance of the equalizer in GSM .In this section, we will reveal the performance of the proposed continuous rate adaptation algorithm and compared with a existing FDSS algorithm which is a blend of high- and low-mobility users in realistic LTE exploitation scenarios.

In Fig-3 the graph is shown for proposed algorithm which is a mixture of low and high mobility users. Proportional Fairness $(\mathrm{PF})$ is a scheduling algorithm which is used to maximize the system throughput, and give at least a minimal level of service to all the users. The Fig-4 is shown for SNR Vs Throughput. Throughput refers to how much data can be transferred from one location to another in a given amount of time. SNR is defined as the ratio of signal power to the noise power, often expressed in decibels. Signal-to-noise ratio is sometimes used informally to refer to the ratio of useful information to false or irrelevant data in a conversation or exchange. While SNR increases, the throughput is also increased. The throughput is increased and compared with FDSS algorithm.

The fig-5 is shown for SNR Vs BER.SNR has more noise and less noise.The bit error rate or bit error ratio (BER) is the number of bit errors divided by the total number of transferred bits during a studied time interval. BER is a unit less performance measure, often expressed as a percentage. In digital transmission, the number of bit errors is the number of received bits of a data stream over a communication channel that have been altered due to noise, interference, distortion or bit synchronization errors.

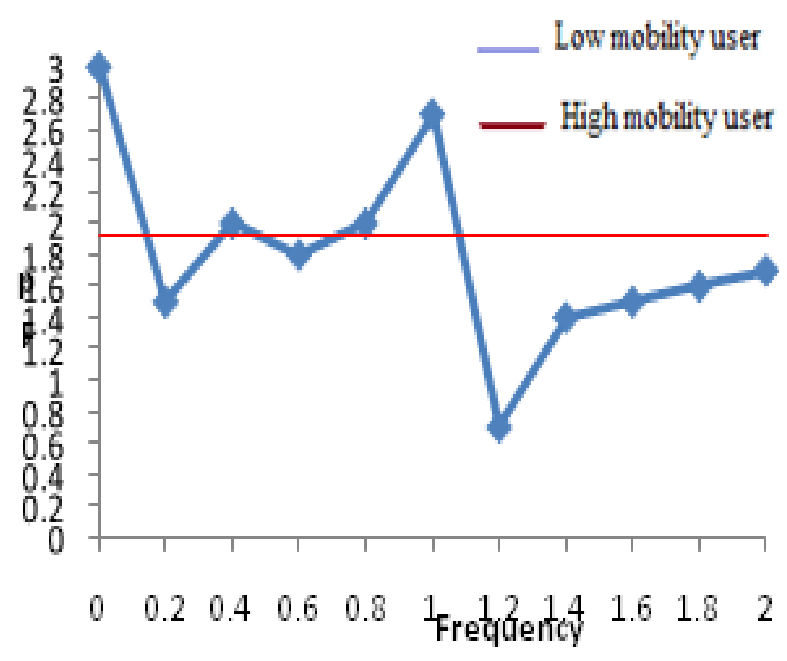

Fig - 3: Frequency Vs Proportional Fairness

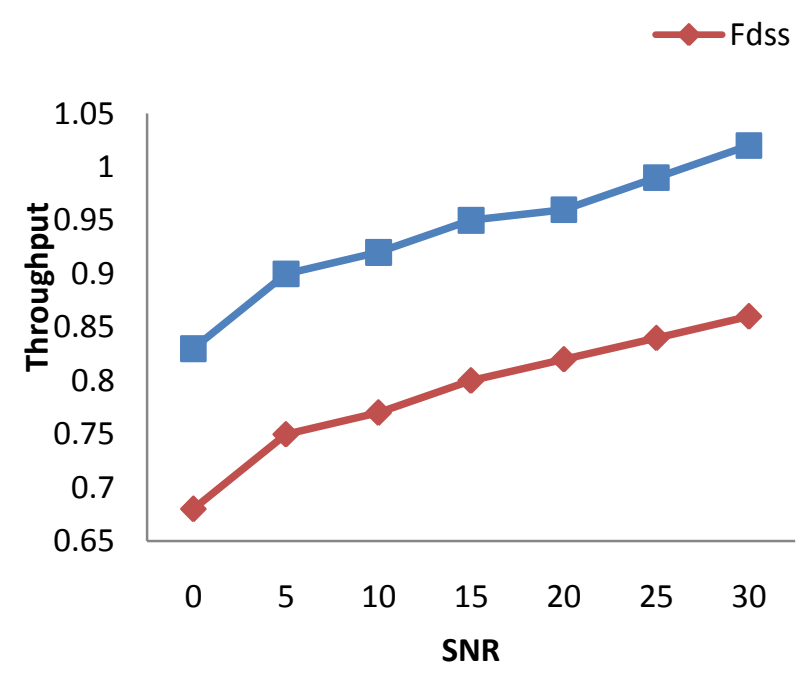

Fig-4: SNR Vs Throughput 


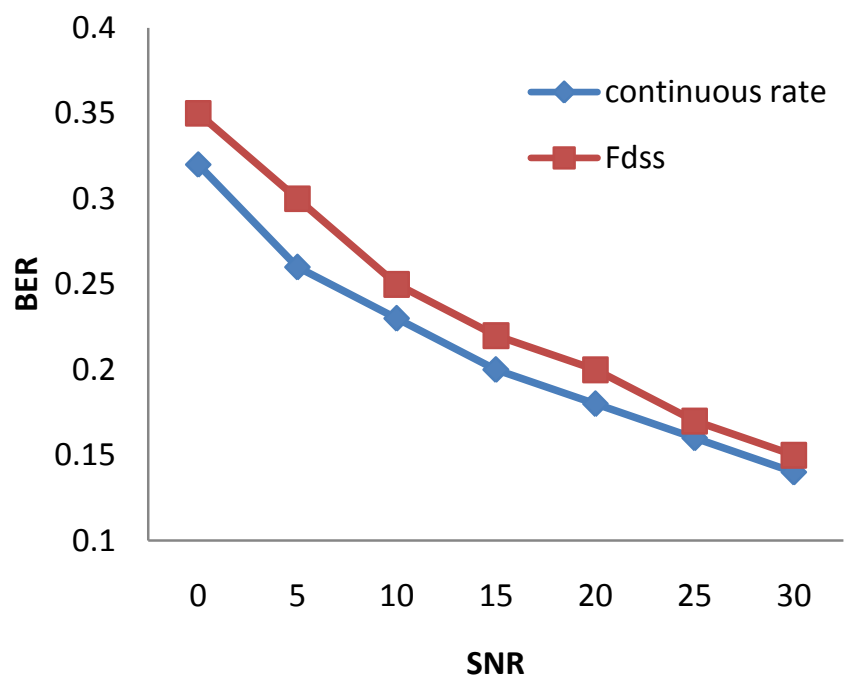

Fig-5: BER Vs SNR

The fig-6 is shown for SNR Vs Capacity. Channel capacity is the tightest upper bound on the rate of information that can be reliably transmitted over a channel. In fig-7 shows spectral efficiency of the capacity. Spectral efficiency is the rate of information which reliably transmits over a communication channel in a given bandwidth. It is a measure of the quantity of users or services that can be simultaneously supported by a limited radio frequency bandwidth

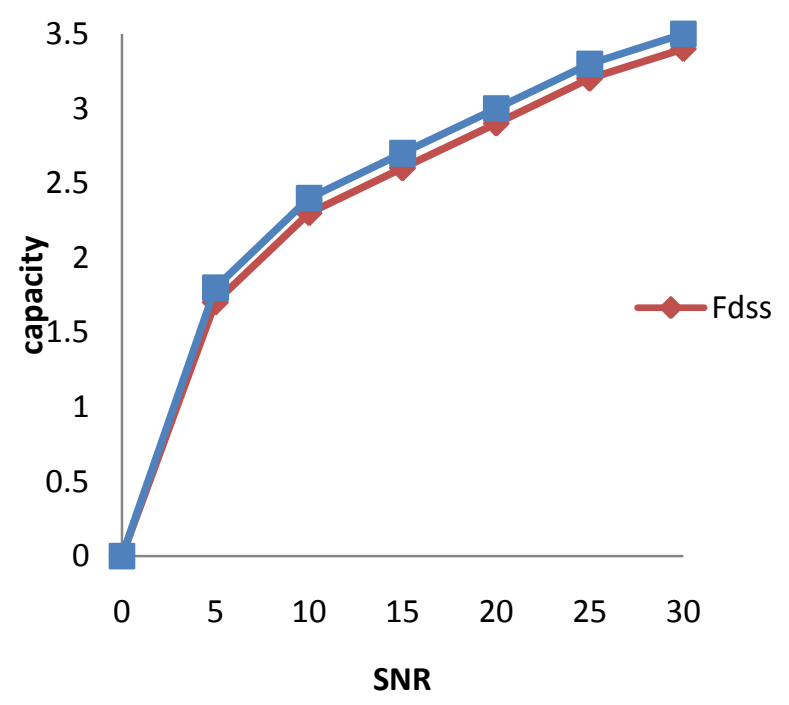

Fig-6: SNR Vs Capacity

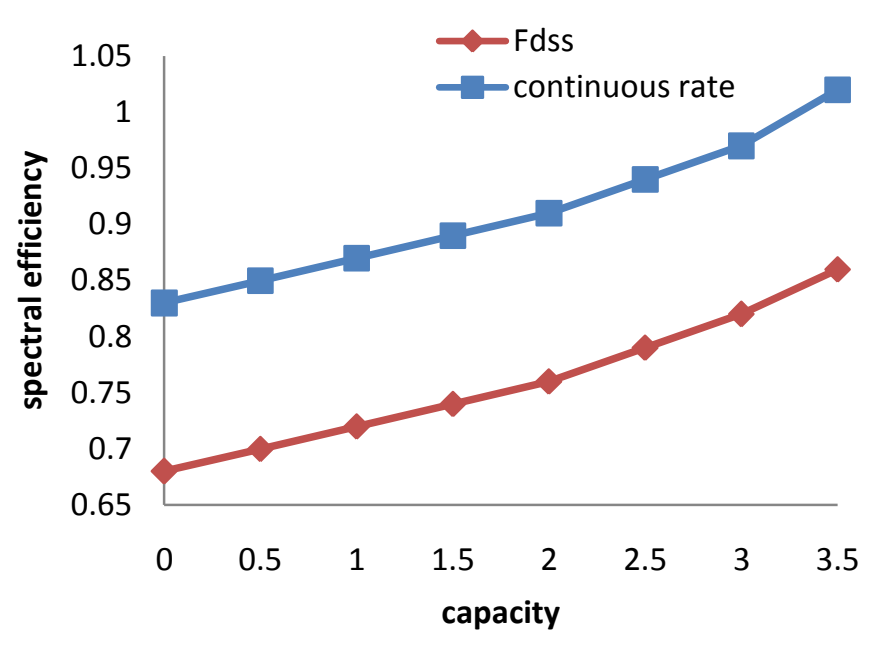

Fig-7: Capacity Vs Spectral Efficiency

\section{CONCLUSIONS}

In this paper we have developed a user classification algorithm. Based on the classification, we propose a scheduling algorithm for different mobilities in LTE downlink systems. The proposed algorithm increases spectral efficiency. The proposed algorithm is compared with frequency diversity selectivity scheduling. The capacity for the mobility users is also determined. In continuous rate adaptation, throughput and capacity is increased when compared to the existing one. The experimental results show that the proposed system results in significant performance improvement.

\section{REFERENCES}

[1] J. Zyren Dr. Wes McCoy, Technical Editor "Overview of the 3gpp long term evolution physical layer" July 2007

[2] F. Capozzi, G.Piro, Student Member, IEEE, L.A. Grieco, Member, IEEE, G. Boggia, Senior Member, IEEE, and P. Camarda. "Downlink Packet Scheduling in LTE Cellular Networks" VOL. 15, NO. 2, 2013

[3] T.Dikamba. "Downlink Scheduling in 3GPP Long Term Evolution (LTE)". MSc Thesis, TUDelft, March 2011

[4] S. Hussain. "Dynamic radio resource management in 3GPP LTE”Blekinge Institute of Technology 2009.

[5] J. Fan, Q. Yin, G. Y. Li, B. Peng, and X. Zhu, "Adaptive block- level resource allocation in OFDMA networks," in IEEE Trans. Wireless Commun., vol. 10,, pp. 396639722011.

[6] H. Kim and Y. Han, "A proportional fair scheduling for multicarrier transmission systems," IEEE Commun. Lett, vol. 9, no. 3, pp. 210-212 Mar. 2005.

[7] D. Calabuig, J. F. Monserrat, D. Gomez- Barquero and O. Lazaro, "An Efficient Dynamic Resource Allocation Algorithm for Packet Switched Communication 
Networks Based on Hopfield Neural Excitation Method".

[8] A. Mills, D. Lister, M. D.Vos, and Y. Ji, "The impact of MS velocity on the performance of frequency selective scheduling in IEEE 802.16 Mobile WiMAX," in 2010 IEEE Consumer Commun. And Networking Conf., pp. $1-5$.

[9] S. J. Lee, "Trade-off between frequency diversity gain and frequency Selective scheduling gain in OFDMA systems in spatial diversity, IEEE Commun. Lett, vol. 11, no. 6, pp. 507-509, Jun. 2007.

[10] R. Kwan, Cyril Leung, and Jie Zhang "Multiuser scheduling on the downlink of an LTE cellular system, "Research Lett. In Commun, vol. 2008, pp. 1-3, Jan. 2008.

[11] M. H. Habaebi, j. chebil, a.g. al- sakkaf and t. h. dahawi "Comparison between scheduling techniques in long term evolution" IEEE Transactions on Vehicular Technology, Vol. 59, No. 9, November 2010

[12] T.Rapperport "Wireless Communication: Principles and Practice Prentice Hall ,2002

[13] J.Huang, Vijay Subramanian, R. Berry, and R. Agrawal "Scheduling and Resource Allocation in OFDMA Wireless Systems" February 2009.

[14] T. Girici,,Jonathan R. Agre, and Anthony "Proportional Fair Scheduling Algorithm OFDMA-Based Wireless Systems with QoS Constraints" Journal of Communications And Networks, Vol. 12, No. 1, February 2010

[15] O. Iosif and I. Banica. " On the Analysis of Packet Scheduling in Downlink 3GPP LTE System" Proceedings of the 4th International Conference on Communication Theory, Reliability and Quality of Service, Budapest, 17-22 April 2011, pp. 99-102..

[16] H. Kim and Y. Han, "A proportional fair scheduling for multicarrier transmission systems," IEEE Commun. Lett, vol. 9, no. 3, pp. 210-212 Mar. 2005.

[17] J. Chan and A. Seneviratne, "A practical user mobility prediction algorithm for supporting adaptive qos in wireless networks," IEEE International Conf. on Networks, pp. 104-111 1999.

[18] International Telecommunication Union, "Guidelines for evaluation of radio interface technologies for IMTAdvanced," ITU-R, Report M.2135-1, 2010

[19] S. N. Donthi and N. B. Mehta, "Joint performance analysis of channel quality indicator feedback schemes and frequency-domain scheduling for LTE," IEEE Trans. Veh. Technol., vol. 60, no. 7, pp. 3096-3109, Sep. 2011.

[20] Varanese, N.; Vicario, J.L.; Spagnolini, U.,’On the Asymptotic Throughput of OFDMA Systems with Best M CQI Feedback, "Wireless Communications Letters, IEEE, vol.1, no.3, pp.145,148, June 2012.

[21] J. Huang, V.G. Subramanian, R. Agrawal, R.A. Berry,"Downlink scheduling and resource allocation for ofdm systems," Wireless Communications, IEEE Transactions on, vol. 8, no. 1, pp. 288 -296, Jan. 2009.

[22] E. Dahlman, S. Parkvall, J. Skold, and P. Beming, 3G Evolution, Second Edition: HSPA and LTE for Mobile Broadband. Academic Press, 2008. 